\title{
Clinical Course and Risk Factors for Liver Injury of Severe and Critical Patients With COVID-19: A Single-centered, Retrospective, Observational Study
}

Jingyuan Liu

Capital Medical University Affiliated Beijing Ditan Hospital

Chunjing Du

Capital Medical University Affiliated Beijing Ditan Hospital

Siyuan Yang

Capital Medical University Affiliated Beijing Ditan Hospital

Lin Pu

Capital Medical University Affiliated Beijing Ditan Hospital

Pan Xiang

Capital Medical University Affiliated Beijing Ditan Hospital

Ang Li ( $\sim$ liang@ccmu.edu.cn )

Department of Critical Care Medicine, Beijing Ditan Hospital, Capital Medical University, Beijing, P. R. China https://orcid.org/0000-0002-1478-3072

\section{Research}

Keywords: Coronavirus disease 2019 (COVID-19), Severe acute respiratory syndrome coronavirus 2 (SARS-CoV-2), Abnormal liver tests, Liver injury, Pneumonia

Posted Date: September 2nd, 2020

DOl: https://doi.org/10.21203/rs.3.rs-66769/v1

License: (a) (1) This work is licensed under a Creative Commons Attribution 4.0 International License. Read Full License 
1 Clinical course and risk factors for liver injury of severe and critical

2 patients with COVID-19: a single-centered, retrospective,

3 observational study

4 Jingyuan $\mathrm{Liu}^{1 *}$, Chunjing $\mathrm{Du}^{1 *}$, Siyuan Yang ${ }^{2 *}, \mathrm{Lin} \mathrm{Pu}^{1}$, Pan Xiang ${ }^{1}$, Ang $\mathrm{Li}^{1 /}$

$6{ }^{1}$ Department of Critical Care Medicine, Beijing Ditan Hospital, Capital Medical

7 University, Beijing, P. R. China

$8{ }^{2}$ Laboratory of Infectious Diseases Center, Beijing Ditan Hospital, Capital Medical

9 University, Beijing, P. R. China

$11 *$ These authors contributed equally to this work.

\#Corresponding author:

Prof. Ang Li

Department of Critical Care Medicine, Beijing Ditan Hospital, Capital Medical

University.

Postal address: Beijing Ditan Hospital, No. 8 Jingshundong Street, Chaoyang District,

Beijing 100015, China.

E-mail: liang@,ccmu.edu.cn

Tel.: 00861084320001

Fax: 00861084322606 


\section{Abstract}

Background: The information regarding the clinical course of COVID-19 patients with liver injury is very limited, especially in severe and critical patients. The objective of this study was to describe the characteristics and clinical course of patients admitted with severe and/or critical SARS-CoV-2 infection in liver function, as well as explore the risk factors that affect liver function in the enrolled COVID-19 patients.

Methods: Information on clinical characteristics of 63 severe and critical patients with confirmed COVID-19 were collected and analyzed.

Results: The incidence of abnormal aspartate aminotransferase, alanine aminotransferase, and total bilirubin in the critical group was obviously higher than in the severe group $(81.48 \%, 81.49 \%, 62.67 \%$, and $45.71 \%, 63.88 \%, 22.86 \%$, respectively, $\mathrm{p}<0.05$ ). The time for liver parameters to reach their peak or trough was approximately 2-3 weeks. No significant difference was observed in cycle threshold values of open reading frame $1 \mathrm{ab}$ and nucleocapsid protein gene on admission or at the peak among liver injury group, abnormal group and normal group $(\mathrm{p}>0.05)$. Patients with invasive ventilator, decreased percentage of neutrophil, lymphocyte and monocyte, and SOFA score $\geq 2(\mathrm{p}<0.05)$ were the independent factors associated with liver injury.

Conclusions: Abnormal liver tests are commonly observed in severe and critical patients with COVID-19. The time of 2-3 weeks after admission should be paid attention to patients with critical COVID-19 in case of the occurrence of liver injury. As independent risk factors for the occurrence of liver injury, regarding decreased ratio of neutrophils, lymphocytes and monocytes, the requirement of invasive ventilator, and SOFA score $\geq 2$, patients with these abnormal parameters should be of 
particular concerned during hospitalization.

Keywords: Coronavirus disease 2019 (COVID-19) · Severe acute respiratory syndrome coronavirus $2(\mathrm{SARS}-\mathrm{CoV}-2) \cdot$ Abnormal liver tests $\cdot$ Liver injury $\cdot$ Pneumonia

\section{Background}

The pandemic of coronavirus disease 2019 (COVID-19), caused by severe acute respiratory syndrome coronavirus 2 (SARS-CoV-2), poses an unprecedented threat to public health[1-3]. Patients with COVID-19 are frequently associated with pulmonary lesions, however, increasing data revealed that COVID-19 has systemic manifestations affecting multiorgan system including liver injury, myocarditis, thrombosis, and coagulation[4-7]. While the impact of COVID-19 on the liver remains unclear, a considerable proportion of patients with elevated liver enzyme have been reported ${ }^{[7-12]}$. Some studies found a mild [1-2 times the upper limit of normal (ULN)] increase in transaminases, while severe liver injury also has been reported[6,13].

However, the information regarding the clinical course of COVID-19 patients with liver injury is very limited, especially in severe and critical patients. Knowledge of the clinical characteristics of liver injury in this disease is vital to answering questions about the therapy and management for patients infected with SARS-CoV-2. Thus, we present a detailed characteristics and clinical course of patients admitted with severe and/or critical SARS-CoV-2 infection in liver function and further to explore the independent risk factors that affect liver function in enrolled patients. 


\section{Methods}

\section{Study design}

This retrospective study included 63 severe and critical patients with confirmed COVID-19 hospitalized in Beijing Ditan Hospital from January $20^{\text {th }}, 2020$ to April $06^{\text {th }}, 2020$. The diagnosis and severity assessment of COVID-19 were defined in accordance with Chinese management guideline for COVID-19 (version 7) released by National Health Commission of China. Patients meeting any one of the following should be considered as severe cases: oxygen saturation at rest $\leqslant 93 \%$ on room air; respiratory distress, or respiratory rate $\geq 30$ breaths/min. Patients who occurred acute respiratory failure $\left(\mathrm{PaO}_{2} / \mathrm{FiO}_{2}<300,30>\right.$ breaths/min $)$, shock, or any organ failure that required mechanical ventilation or intensive care management should be considered as critical cases. Acute respiratory distress syndrome (ARDS) was defined according to Berlin definition[14]. Cases were excluded for patients who were younger than 18 years, as well as mild and moderate ill patients. This study was approved by the Institutional Review Board of Capital Medical University affiliated Beijing Ditan Hospital and patient-level informed consent was waived owing to its retrospective nature.

\section{Data collection}

The medical records of 63 patients were collected by the research team. Data on patients' demographics, comorbidities, vital signs, laboratory characteristics and treatment were acquired by the hospitalization management system.

\section{Laboratory examination and Liver test parameters}


On admission, laboratory parameters including peripheral leukocyte count, neutrophils, lymphocytes, monocytes, platelets, haemoglobin, hematocrit, C-reactive protein (CRP), International Normalized Ratio (INR), D-dimer, creatine kinase, aspartate aminotransferase (AST), alanine transaminase (ALT), total bilirubin (TBIL), serum albumin, and A/G (albumin/ globulin) ratio were collected. Since COVID-19 is an emerging infectious disease, consensus or guidance on the classifications of liver injury are lacking, we classified the pattern of liver test into three groups: normal liver test, abnormal liver test, and liver injury. Abnormal liver test was defined as the elevation of serum liver enzymes exceeding the upper limit of normal (ULN), that is, AST $>40 \mathrm{U} / \mathrm{L}, \mathrm{ALT}>40 \mathrm{U} / \mathrm{L}$, and TBIL $>17.1 \mathrm{umol} / \mathrm{L}$. Liver injury was defined when ALT and/or AST over $3 \times \mathrm{ULN}$, and/or TBIL over $3 \times$ UL. Furthermore, the dynamic changes of liver enzymes, serum albumin, INR, and A/G ratio were also recorded.

\section{SARS-CoV-2 RNA Detection}

COVID-19 was diagnosed according to the cycle threshold $(\mathrm{Ct})$ values of open reading frame $1 \mathrm{ab}(\mathrm{ORF} 1 \mathrm{ab})$ and nucleocapsid protein $(\mathrm{N})$ gene by RT-PCR assay. The assay was performed by the National Center for Disease Control or the Clinical Laboratory of Beijing Ditan hospital using a commercial kit (Daan, Guangzhou, China). The SARS-CoV-2 viral loads were measured by the copy number of the $\mathrm{N}$ gene from sputum samples or throat swabs of the COVID-19 cases. Ct values were negatively correlated with viral RNA copy numbers[15]. According to the instruction of RT-PCR kit, patients with Ct value less than 40 was considered as positive.

\section{Statistical analysis}


All statistical analyses were performed with SPSS 19.0 for Windows (IBM, USA).

Continuous variables were described as mean and SD or median and IQR, and categorical variables as frequency and percentages. Normally distributed variables were analyzed using independent group t-test or one-way ANOVA, whereas the Mann-Whitney nonparametric were used for non-normally distributed variables.

Categorical variables were conducted using the chi-square $(\chi 2)$ or Fisher's exact tests. Pearson's correlation coefficient was performed to assess the correlation between the severity of liver injury and laboratory results. Ordinal logistic regression analysis was conducted to evaluate the association of baseline characteristics with the severity of liver injury. Two-sided $\mathrm{P}$ values of less than 0.05 were considered statistically significant.

\section{Results}

\section{Baseline characteristics of enrolled patients with COVID-19}

The clinical characteristics of enrolled patients with COVID-19 are shown in Table 1. A total of 63 patients were enrolled in the study. The average age of 63 patients was 56.75 years and 41 patients $(65.08 \%)$ were male. According to the severity of the disease, subjects were classified as severe group (36, 57.14\%) and critical group (27, $42.86 \%$ ). There was significant difference between two groups in oxygen therapy and drug use except the requirement of high flow oxygen or non-invasive ventilator. In terms of laboratory results, blood indices, including peripheral white cell count, neutrophil, C-reactive protein (CRP), BUN, and LDH (lactate dehydrogenase), in critical patients were significantly higher than in severe patients $(\mathrm{p}<0.05)$.

Contrastingly, lymphocyte, percentage of monocyte, haemoglobin, and hematocrit in critical patients were significantly lower than in severe patients $(\mathrm{p}<0.05)$. 


\section{Clinical features of enrolled patients with COVID-19 and liver function tests} during hospitalization

The serum liver enzyme parameters of enrolled patients were further analyzed. Results showed that there was no difference between both groups in AST, ALT, TBIL, INR, albumin, and A/G ratio on admission (Supplemental Table), whereas the peak values (or trough values) of these parameters have significant differences except ALT (Table 2, Figure 1a,b). The incidence of abnormal AST, ALT, and TBIL in the critical group was obviously higher than in the severe group $(81.48 \%, 81.49 \%$, $62.67 \%$, and $45.71 \%, 63.88 \%, 22.86 \%$, respectively, $\mathrm{p}<0.05$ ) during hospitalization. Based on the test of liver function, subjects were further classified as normal liver test (11, 17.46\%), abnormal liver test (32, 50.79\%), and liver injury group (20,31.75\%)

(Table 3). Patients in the three groups were not significantly different in sex ratio and age. The CRP, percentage of neutrophil, BUN, and LDH in liver injury group were significantly higher than in non-liver group, while the percentage of lymphocyte and monocyte were on the contrary $(\mathrm{p}<0.05)$. Additionally, the oxygenation index of liver injury group was significantly lower than that of the other groups $(\mathrm{p}=0.015)$, while the CURB-65 score, SOFA score, the incidence of ARDS, and the requirement of invasive ventilator were significantly higher in the liver injury group than in the non-livery group $(\mathrm{p}<0.05)$ (Table 3, Figure $1 \mathrm{c}-\mathrm{f})$.

\section{Dynamic profile of liver function indicators and viral clearance}

To determine the dynamic changes of enrolled patients in liver function parameters and virus clearence, data of liver enzymes and $\mathrm{Ct}$ values were recorded during hospitalization. Results showed that the level of AST,TBIL, and INR was significant 
higher in the critical group than in the severe group and the time for these indicators to reach their peak was approximately 2-3 weeks (Figure 2a-b, e). Compared with AST, the duration of TBIL was longer, which reached its peak at about 4 weeks, then gradually decreased. Conversely, the level of ALT, albumin and A/G ratio, was significant lower in the critical group than in the severe group, the time for these parameters to reach their trough was approximately 2-3 weeks as well (Figure 2b-c, f). As shown in Supplemental Figure 1a-f, the liver injury group has similar characteristics. Despite the level of Ct values in the critical group was higher than in the severe group (Figure 3a), there was no significant difference on admission or at the peak between severe group and critical group, as well as among liver injury group, abnormal group and normal group (Supplemental Figure 2a-d). The Ct values of patients with different groups decreased gradually during hospitalization and the time of virus clearance was approximately 2-3 weeks after admission (Figure 3a-b).

\section{Independent factors associated with severity of liver function}

We further to assess the correlation between the severity of liver injury and laboratory results, as well as the independent factors associated with liver tests of abnormality or injury. As shown in Figure 4a-f, the severity of liver function were positively correlated with percentage of neutrophil, CRP, CURB-65 score and SOFA score, and negatively correlated with percentage of lymphocyte, percentage of monocyte (data were not shown), and oxygenation index. Variables with p-values of $<0.05$ in univariable analysis were included in the ordinal logistic analysis to identify the significant indicators affecting liver function in enrolled COVID-19 patients (Table 4). Results revealed that SOFA score $\geq 2[\mathrm{OR}=165.41,95 \%$ confidence interval $(\mathrm{CI})=$ $(1.57,8.64) ; \mathrm{p}=0.005]$ were factors positively associated with abnormality or injury 
as indicated by liver tests. After adjustment for age, sex, and comorbidities, patients with invasive ventilator, the decreased percentage of neutrophil, lymphocyte and monocyte, and SOFA score $\geq 2$ were the independent factors associated with liver tests of abnormality or injury.

\section{Discussion}

In this cohort with 63 cases, we demonstrate that abnormal liver tests are common in patients with severe and critical COVID-19. Patients with critical COVID-19 should be aware of the occurrence of liver injury in 2-3 weeks after admission. Particular attention should be paid to patients with decreased ratios of neutrophils, lymphocytes and monocytes, the requirement of invasive ventilator, and SOFA score $\geq 2$ during hospitalization, as these are independent risk factors for the occurrence of liver injury.

The occurrence of liver enzyme elevation observed here is ranged from $22.86 \%$ to $81.49 \%$ in severe and critical patients during hospitalization, which is higher than previous studies ranged from $14 \%$ to $53 \%$ in patients including mildly and moderate COVID-19[16], mainly manifested by the elevated levels of ALT AST and TBIL accompanied by the slightly decreased albumin levels. Indeed, the increased liver enzymes were observed more commonly in the critical group than in the severe group. Interestingly, we observed that the levels of AST and TBIL were more higher in critical group than in severe group, while no difference were observed between both groups in ALT. Considering the elevated AST could be from muscle damage rather than directly reflecting liver injury and the levels of INR were primarily within the range of 1.5 in this study, the discovery that SARS-CoV-2 virus bind to angiotensin- 
converting enzyme 2 (ACE2) on hepatocytes, especially on biliary epithelial cells, then cause liver injury[17] may partially explain the results in our patients. In other words, patients with liver injury in COVID-19 were more likely to cholestatic type rather than hepatocellular type.

Currently, the underlying mechanisms of COVID-19 related liver injury remain unclear. In fact, it may be multifactorial and individualized. First, a hyperinflammatory response to COVID-19 may contribute to liver injury[11,18,19]. Evidence is that the severity of liver function were positively correlated with the percentage of neutrophil and CRP, as well as negatively correlated with percentage of lymphocyte and monocyte, meanwhile, the decreased ratios of neutrophils, lymphocytes and monocytes are independent risk factors for the occurrence of liver injury. Hepatic inflammation involving activation of innate immune system accompanied by the cytokine storm is a well-established driver of liver injury[20]. Notably, lymphopenia was commonly observed in COVID-19 studies and patients with lower counts of lymphocyte are more susceptible to fatal outcomes[21]. Second, whether SARS-CoV-2 can directly infect hepatocytes remain undetermined[19,22]. In present study, no significant difference was observed in $\mathrm{Ct}$ values on admission or at the peak among liver injury group, abnormal group and normal group. However, it is impossible to demonstrated whether the virus has an impact on liver cytopathy owing to the lack of liver biopsy and Ct values of the liver in situ. ACE2 is abundantly expressed in the hepatocytes, especially in biliary epithelial cells, and the liver may be a potential target for direct infection [17], that was however not yet demonstrated. Thus, the influence of SARS-CoV-2 on liver begs for further investigation. Third, hypoxia induced by COVID-19-related complications (i.e., acute respiratory distress 
syndrome and multiple organ failure) may also induce hepatic ischemia and hypoxiareperfusion dysfunction[23,24]. Evidence is that the severity of liver function were positively correlated with CURB-65 score and SOFA score, and negatively correlated with oxygenation index, meanwhile, the requirement of invasive ventilator and SOFA score $\geq 2$ are independent risk factors for the occurrence of liver injury. Lastly, drugelicited liver injury may also account for laboratory test abnormalities[25,26]. However, there was no significant difference in drug use among liver injury group, abnormal group and normal group in this study, except the vasoactive drug. Besides, the vasoactive drug wasn't independent risk factor for the occurrence of liver injury in the logistic analysis. Thus, the enrolled drugs in this study may not directly induce liver injury.

Our results showed the time for liver parameters to reach their peak or trough was approximately 2-3 weeks, which is critical for clinical implication in management for patients infected with SARS-CoV-2. Thus, regular monitoring of liver function tests should be performed, particularly in patients with severe and critical COVID-19.

This study has some limitations. First, not all laboratory tests were collected in enrolled patients, including alkaline phosphatase, and gamma-glutamyl transferase owing to the retrospective design. Additionally, it is impossible to demonstrated whether the virus has an impact on liver cytopathy owing to the lack of liver biopsy and viral loads results of the liver in situ. Further studies should corroborate the pathogenic mechanism. Meanwhile, the relatively small sample size also a limitation of this study. Future studies are needed to enroll a larger sample sizes to strengthen the accuracy of the results. 


\section{Conclusion}

In summary, abnormal liver tests are commonly observed in severe and critical patients with COVID-19. The time of 2-3 weeks after admission should be paid attention to patients with critical COVID-19 in case of the occurrence of liver injury. As independent risk factors for the occurrence of liver damage, regarding decreased ratio of neutrophils, lymphocytes and monocytes, the requirement of invasive ventilator, and SOFA score $\geq 2$, patients with these abnormal parameters should be of particular concerned during hospitalization.

\section{Abbreviations}

COVID-19, coronavirus disease 2019; SARS-CoV-2, severe acute respiratory syndrome coronavirus 2; ALT, alanine aminotransferase; AST, aspartate Assessment; NLR = neutrophil to lymphocyte ratio; $\mathrm{CRP}=\mathrm{C}$-reactive protein; BUN

= blood urea nitrogen; $\mathrm{A} / \mathrm{G}=$ albumin/ globulin; INR = international normalized ratio;

$\mathrm{LDH}=$ lactate dehydrogenase $\mathrm{Ct}=$ cycle threshold

\section{Acknowledgments}

291 The authors gratefully acknowledge Gang Wan Ph.D. and Junnan Li Ph.D. for their assistance with data analysis. Thanks to all the front-line medical staff of Beijing Ditan 
hospital for their bravery and efforts in SARS-CoV-2 prevention and control.

294 Jingyuan Liu ${ }^{1 *}$, Chunjing Du ${ }^{1 *}$, Siyuan Yang ${ }^{2 *}, \mathrm{Lin} \mathrm{Pu}^{1}$, Pan Xiang ${ }^{1}, \mathrm{Ang} \mathrm{Li}^{1 \text { \# }}$

\section{Authors' contributions}

Jingyuan Liu, Chunjing Du and Siyuan Yang collected the clinical data. Lin Pu, Pan Xiang and Ang Liinterpreted the data. Chunjing Du and wrote the manuscript. Jingyuan Liu proofread the manuscript. Ang Li calculated the statistics and proofread the manuscript. All authors read and approved the final manuscript.

Funding: This work was supported by Beijing Municipal Science and technology commission, China [Grant No: Z201100005420012].

\section{Availability of data and materials}

The datasets used and/or analyzed during the current study are available from the corresponding author on reasonable request.

\section{Ethics approval and consent to participate}

This study was approved by the Institutional Review Board of Capital Medical

University affiliated Beijing Ditan Hospital and patient-level informed consent was waived owing to its retrospective nature. The study was performed in accordance with the ethical standards laid down in the 1964 Declaration of Helsinki and its later amendments.

\section{Consent for publication}




\section{Competing interests}

The authors declare that they have no competing interests.

321

322

\section{References:}

1 Huang C, Wang Y, Li X, Ren L, Zhao J, Hu Y, Zhang L, Fan G, Xu J, Gu X, Cheng Z, Yu T, Xia J, Wei Y, Wu W, Xie X, Yin W, Li H, Liu M, Xiao Y, Gao H, Guo L, Xie J, Wang G, Jiang R, Gao Z, Jin Q, Wang J, Cao B: Clinical features of patients infected with 2019 novel coronavirus in Wuhan, China. The Lancet 2020;395:497-506.

2 Richardson S, Hirsch JS, Narasimhan M, Crawford JM, McGinn T, Davidson KW, Barnaby DP, Becker LB, Chelico JD, Cohen SL, Cookingham J, Coppa K, Diefenbach MA, Dominello AJ, DuerHefele J, Falzon L, Gitlin J, Hajizadeh N, Harvin TG, Hirschwerk DA, Kim EJ, Kozel ZM, Marrast LM, Mogavero JN, Osorio GA, Qiu M, Zanos TP: Presenting characteristics, comorbidities, and outcomes among 5700 patients hospitalized with COVID-19 in the new york city area. JAMA 2020

3 Grasselli G, Zangrillo A, Zanella A, Antonelli M, Cabrini L, Castelli A, Cereda D, Coluccello A, Foti G, Fumagalli R, Iotti G, Latronico N, Lorini L, Merler S, Natalini G, Piatti A, Ranieri MV, Scandroglio AM, Storti E, Cecconi M, Pesenti A: Baseline characteristics and outcomes of 1591 patients infected with SARS-CoV-2 admitted to ICUs of the lombardy region, italy. JAMA 2020;323:1574.

4 Xie H, Zhao J, Lian N, Lin S, Xie Q, Zhuo H: Clinical characteristics of non-ICU hospitalized patients with coronavirus disease 2019 and liver injury: A retrospective study. LIVER INT 2020;40:1321-1326.

5 Zhou F, Yu T, Du R, Fan G, Liu Y, Liu Z, Xiang J, Wang Y, Song B, Gu X, Guan L, Wei Y, Li H, Wu X, Xu J, Tu S, Zhang Y, Chen H, Cao B: Clinical course and risk factors for mortality of adult inpatients with COVID-19 in Wuhan, China: A retrospective cohort study. LANCET 2020;395:10541062 .

6 Wang D, Hu B, Hu C, Zhu F, Liu X, Zhang J, Wang B, Xiang H, Cheng Z, Xiong Y, Zhao Y, Li Y, Wang X, Peng Z: Clinical Characteristics of 138 Hospitalized Patients with 2019 Novel CoronavirusInfected Pneumonia in Wuhan, China. JAMA 2020

7 Chen N, Zhou M, Dong X, Qu J, Gong F, Han Y, Qiu Y, Wang J, Liu Y, Wei Y, Xia J, Yu T, Zhang X, Zhang L: Epidemiological and clinical characteristics of 99 cases of 2019 novel coronavirus pneumonia in Wuhan, China: A descriptive study. LANCET 2020;395:507-513.

8 Zhang C, Shi L, Wang FS: Liver injury in COVID-19: Management and challenges. Lancet Gastroenterol Hepatol 2020;5:428-430.

9 Cai Q, Huang D, Yu H, Zhu Z, Xia Z, Su Y, Li Z, Zhou G, Gou J, Qu J, Sun Y, Liu Y, He Q, Chen J, Liu L, Xu L: COVID-19: Abnormal liver function tests. J HEPATOL 2020

10 Xu L, Liu J, Lu M, Yang D, Zheng X: Liver injury during highly pathogenic human coronavirus infections. LIVER INT 2020;40:998-1004.

11 Lei F, Liu YM, Zhou F, Qin JJ, Zhang P, Zhu L, Zhang XJ, Cai J, Lin L, Ouyang S, Wang X, Yang 
C, Cheng X, Liu W, Li H, Xie J, Wu B, Luo H, Xiao F, Chen J, Tao L, Cheng G, She ZG, Zhou J, Wang H, Lin J, Luo P, Fu S, Zhou J, Ye P, Xiao B, Mao W, Liu L, Yan Y, Liu L, Chen G, Li H, Huang X, Zhang BH, Yuan Y: Longitudinal association between markers of liver injury and mortality in COVID19 in China. HEPATOLOGY 2020

12 Mantovani A, Beatrice G, Dalbeni A: Coronavirus disease 2019 and prevalence of chronic liver disease: A meta-analysis. LIVER INT 2020;40:1316-1320.

13 Wu C, Chen X, Cai Y, Xia J, Zhou X, Xu S, Huang H, Zhang L, Zhou X, Du C, Zhang Y, Song J, Wang S, Chao Y, Yang Z, Xu J, Zhou X, Chen D, Xiong W, Xu L, Zhou F, Jiang J, Bai C, Zheng J, Song Y: Risk factors associated with acute respiratory distress syndrome and death in patients with coronavirus disease 2019 pneumonia in wuhan, china. JAMA INTERN MED 2020

14 Ranieri VM, Rubenfeld GD, Thompson BT, Ferguson ND, Caldwell E, Fan E, Camporota L, Slutsky AS: Acute respiratory distress syndrome: The Berlin Definition. JAMA 2012;307:2526-2533.

15 Zou L, Ruan F, Huang M, Liang L, Huang H, Hong Z, Yu J, Kang M, Song Y, Xia J, Guo Q, Song T, He J, Yen HL, Peiris M, Wu J: SARS-CoV-2 viral load in upper respiratory specimens of infected patients. N Engl J Med 2020;382:1177-1179.

16 Guan WJ, Ni ZY, Hu Y, Liang WH, Ou CQ, He JX, Liu L, Shan H, Lei CL, Hui D, Du B, Li LJ, Zeng G, Yuen KY, Chen RC, Tang CL, Wang T, Chen PY, Xiang J, Li SY, Wang JL, Liang ZJ, Peng YX, Wei L, Liu Y, Hu YH, Peng P, Wang JM, Liu JY, Chen Z, Li G, Zheng ZJ, Qiu SQ, Luo J, Ye CJ, Zhu SY, Zhong NS: Clinical Characteristics of Coronavirus Disease 2019 in China. N Engl J Med 2020;382:1708-1720.

17 Zhao B, Ni C, Gao R, Wang Y, Yang L, Wei J, Lv T, Liang J, Zhang Q, Xu W, Xie Y, Wang X, Yuan Z, Liang J, Zhang R, Lin X: Recapitulation of SARS-CoV-2 infection and cholangiocyte damage with human liver ductal organoids. PROTEIN CELL 2020

18 Mehta P, McAuley DF, Brown M, Sanchez E, Tattersall RS, Manson JJ: COVID-19: Consider cytokine storm syndromes and immunosuppression. LANCET 2020;395:1033-1034.

19 Li Y, Xiao SY: Hepatic involvement in COVID-19 patients: Pathology, pathogenesis, and clinical implications. J MED VIROL 2020

20 McDonald B, Kubes P: Innate immune cell trafficking and function during sterile inflammation of the liver. GASTROENTEROLOGY 2016;151:1087-1095.

21 Henry BM: COVID-19, ECMO, and lymphopenia: A word of caution. Lancet Respir Med 2020;8:e24.

22 Guan GW, Gao L, Wang JW, Wen XJ, Mao TH, Peng SW, Zhang T, Chen XM, Lu FM: [Exploring the mechanism of liver enzyme abnormalities in patients with novel coronavirus-infected pneumonia]. Zhonghua Gan Zang Bing Za Zhi 2020;28:100-106.

23 Feng G, Zheng KI, Yan QQ, Rios RS, Targher G, Byrne CD, Poucke SV, Liu WY, Zheng MH: COVID-19 and liver dysfunction: Current insights and emergent therapeutic strategies. J Clin Transl Hepatol 2020;8:18-24.

24 Zhang XJ, Cheng X, Yan ZZ, Fang J, Wang X, Wang W, Liu ZY, Shen LJ, Zhang P, Wang PX, Liao R, Ji YX, Wang JY, Tian S, Zhu XY, Zhang Y, Tian RF, Wang L, Ma XL, Huang Z, She ZG, Li H: An ALOX12-12-HETE-GPR31 signaling axis is a key mediator of hepatic ischemia-reperfusion injury. NAT MED 2018;24:73-83.

25 Sun J, Aghemo A, Forner A, Valenti L: COVID-19 and liver disease. LIVER INT 2020;40:12781281.

26 Hajifathalian K, Mahadev S, Schwartz RE, Shah S, Sampath K, Schnoll-Sussman F, Brown RJ, 
Carr-Locke D, Cohen DE, Sharaiha RZ: SARS-COV-2 infection (coronavirus disease 2019) for the gastrointestinal consultant. World J Gastroenterol 2020;26:1546-1553.

\section{Figure legends}

Fig. 1 Caption: The liver function parameters and percentage of liver injury under different groups.

Fig. a: Description text: The peak values of AST, ALT, TBIL, and albumin between the severe group and the critical group.

Fig. b: Description text: The peak values of INR and the trough value of $A / G$ ratio between the severe group and the critical group.

Fig. c: Description text: The percentage of liver injury between CURB-65 score $<2$ and CURB-65 score $\geqslant 2$.

Fig. d: Description text: Tthe percentage of liver injury between SOFA score $<2$ and SOFA score $\geqslant 2$.

Fig. e: Description text: The percentage of liver injury between without ARDS and with ARDS.

Fig. f: Description text: The percentage of liver injury between without ventilator and with ventilator.

Fig. 2 Caption: Dynamic profile of liver function indicators between the severe group and the critical group.

Fig. a: Description text: Dynamic changes of AST between the severe group and the critical group. 
Fig. b: Description text: Dynamic changes of TBIL between the severe group and the critical group.

Fig. c: Description text: Dynamic changes of ALT between the severe group and the critical group.

Fig. d: Description text: Dynamic changes of albumin between the severe group and the critical group.

Fig. e: Description text: Dynamic changes of INR between the severe group and the critical group.

Fig. f: Description text: Dynamic changes of $\mathrm{A} / \mathrm{G}$ ratio between the severe group and the critical group.

Fig. 3 Caption: Dynamic changes of Ct values among differ groups.

Fig. a: Description text: Dynamic changes of $\mathrm{Ct}$ values between the severe group and the critical group.

Fig. b: Description text: Dynamic changes of Ct values among the normal group, the abnormal group, and the liver injury group.

Fig. 4 Caption: the correlation between the severity of liver injury and laboratory results.

Fig. a: Description text: the correlation between the severity of liver injury and the percentage of neutrophil.

Fig. b: Description text: the correlation between the severity of liver injury and CRP.

Fig. c: Description text: the correlation between the severity of liver injury and CURB- 
65 score.

Fig. d: Description text: the correlation between the severity of liver injury and SOFA score.

Fig. e: Description text: the correlation between the severity of liver injury and the percentage of lymphocyte.

Fig. f: Description text: the correlation between the severity of liver injury and oxygenation index.

Supplemental Figure 1 Caption: Dynamic profile of liver function indicators among different groups.

Fig. a: Description text: Dynamic changes of ALT among the normal group, the abnormal group, and the liver injury group.

Fig. b: Description text: Dynamic changes of AST among the normal group, the abnormal group, and the liver injury group.

Fig. c: Description text: Dynamic changes of TBIL among the normal group, the abnormal group, and the liver injury group.

Fig. d: Description text: Dynamic changes of albumin among the normal group, the abnormal group, and the liver injury group.

Fig. e: Description text: Dynamic changes of INR among the normal group, the abnormal group, and the liver injury group.

Fig. f: Description text: Dynamic changes of $A / G$ ratio among the normal group, the abnormal group, and the liver injury group. 
468 Supplemental Figure 2 Caption: the $\mathrm{Ct}$ values under different groups.

469 Fig. a: Description text: the $\mathrm{Ct}$ values on admission between the severe group and the 470 critical group.

471 Fig. b: Description text: the peak values of $\mathrm{Ct}$ values between the severe group and the

472 critical group.

473 Fig. c: Description text: the $\mathrm{Ct}$ values on admission among the normal group, the

474 abnormal group, and the liver injury group.

475 Fig. d: Description text: the peak values of $\mathrm{Ct}$ values among the normal group, the 476 abnormal group, and the liver injury group. 


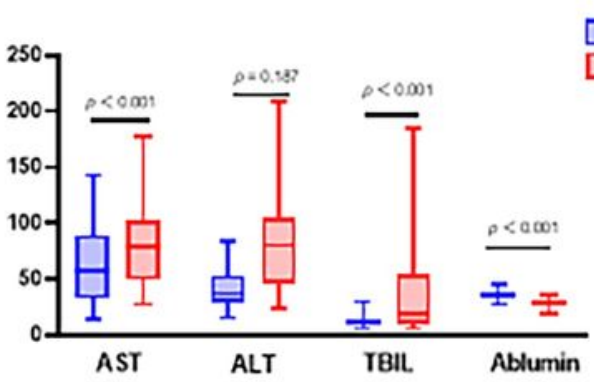

a

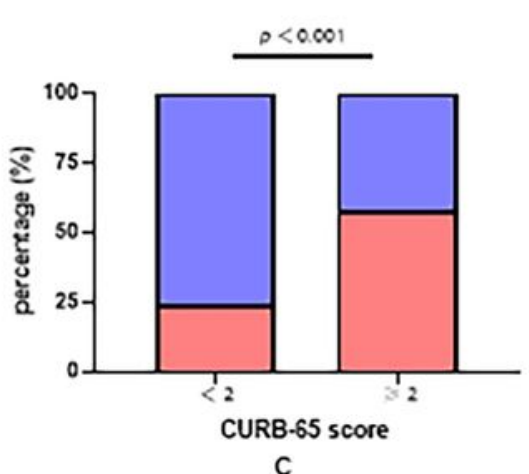

口 Liver inüry

口 Nonliver injuy

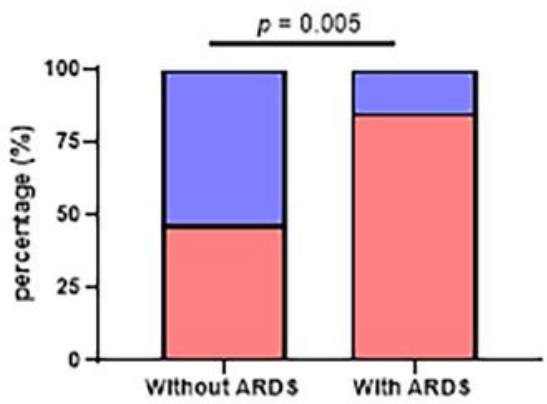

e

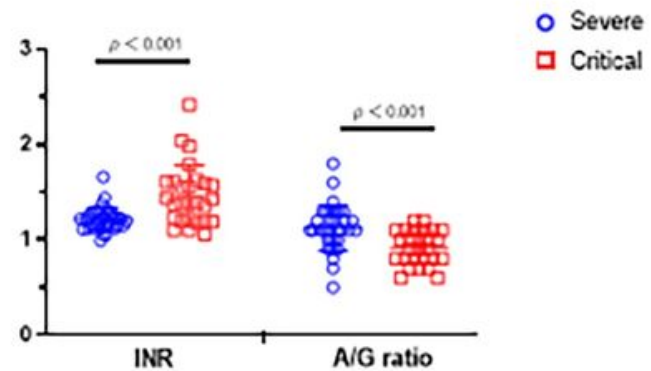

b
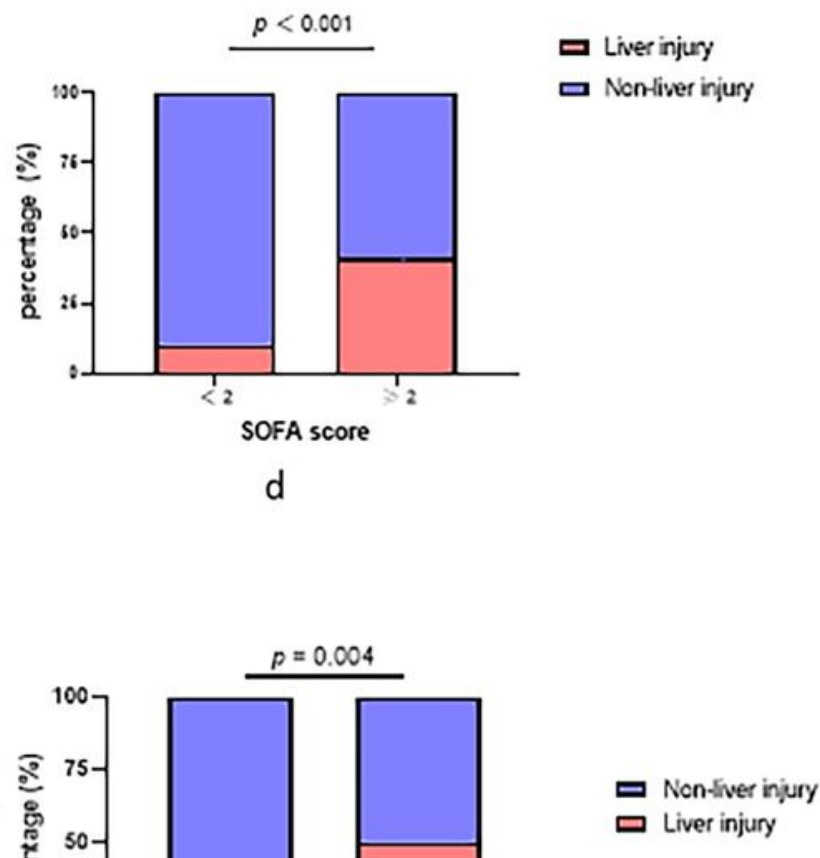

\section{Figure 1}

Caption: The liver function parameters and percentage of liver injury under different groups. Fig. a: Description text: The peak values of AST, ALT, TBIL, and albumin between the severe group and the critical group. Fig. b: Description text: The peak values of INR and the trough value of $A / G$ ratio between 
the severe group and the critical group. Fig. c: Description text: The percentage of liver injury between CURB-65 score $\$ 2$ and CURB-65 score $\geq 2$. Fig. d: Description text: Tthe percentage of liver injury between SOFA score $\mathbb{2}$ and SOFA score $\geq 2$. Fig. e: Description text: The percentage of liver injury between without ARDS and with ARDS. Fig. f: Description text: The percentage of liver injury between without ventilator and with ventilator.
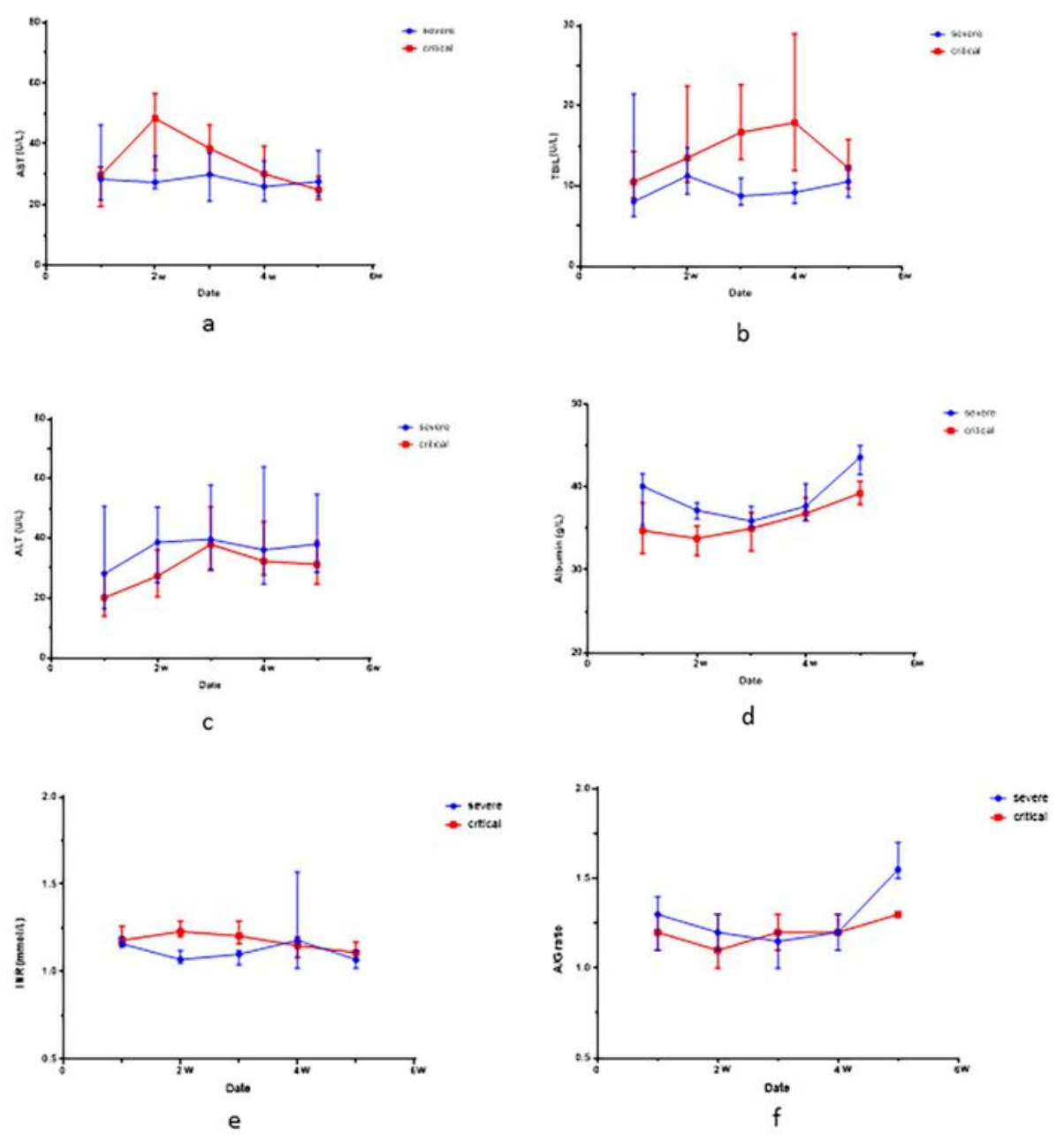

Figure 2 
Caption: Dynamic profile of liver function indicators between the severe group and the critical group. Fig. a: Description text: Dynamic changes of AST between the severe group and the critical group. Fig. b: Description text: Dynamic changes of TBIL between the severe group and the critical group. Fig. c: Description text: Dynamic changes of ALT between the severe group and the critical group. Fig. d: Description text: Dynamic changes of albumin between the severe group and the critical group. Fig. e: Description text: Dynamic changes of INR between the severe group and the critical group. Fig. $\mathrm{f}$ : Description text: Dynamic changes of $A / G$ ratio between the severe group and the critical group.

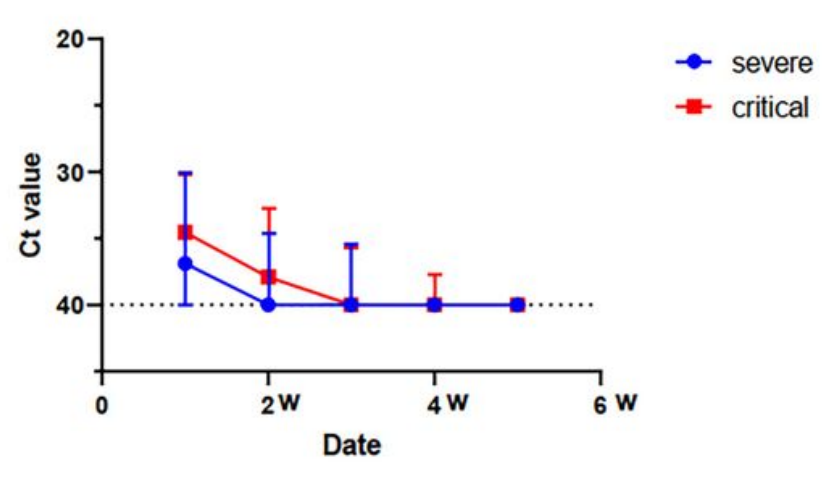

a

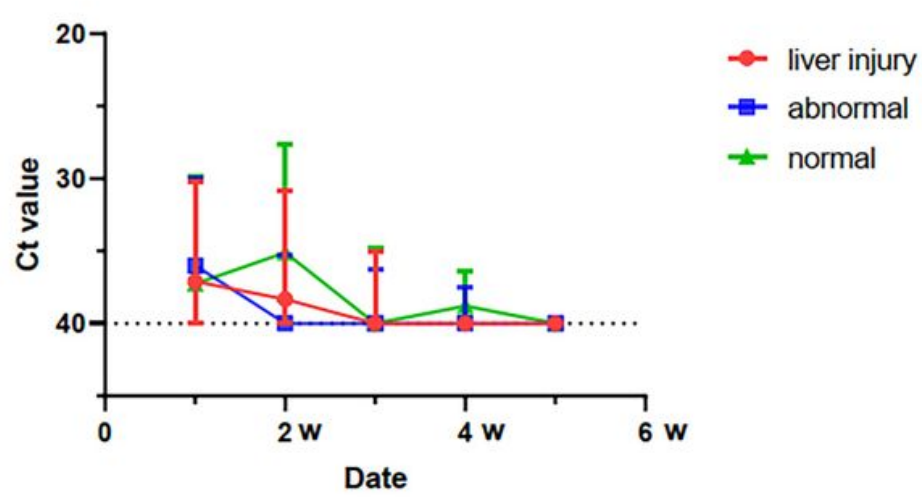

b

\section{Figure 3}

Caption: Dynamic changes of Ct values among differ groups. Fig. a: Description text: Dynamic changes of Ct values between the severe group and the critical group. Fig. b: Description text: Dynamic changes of Ct values among the normal group, the abnormal group, and the liver injury group. 

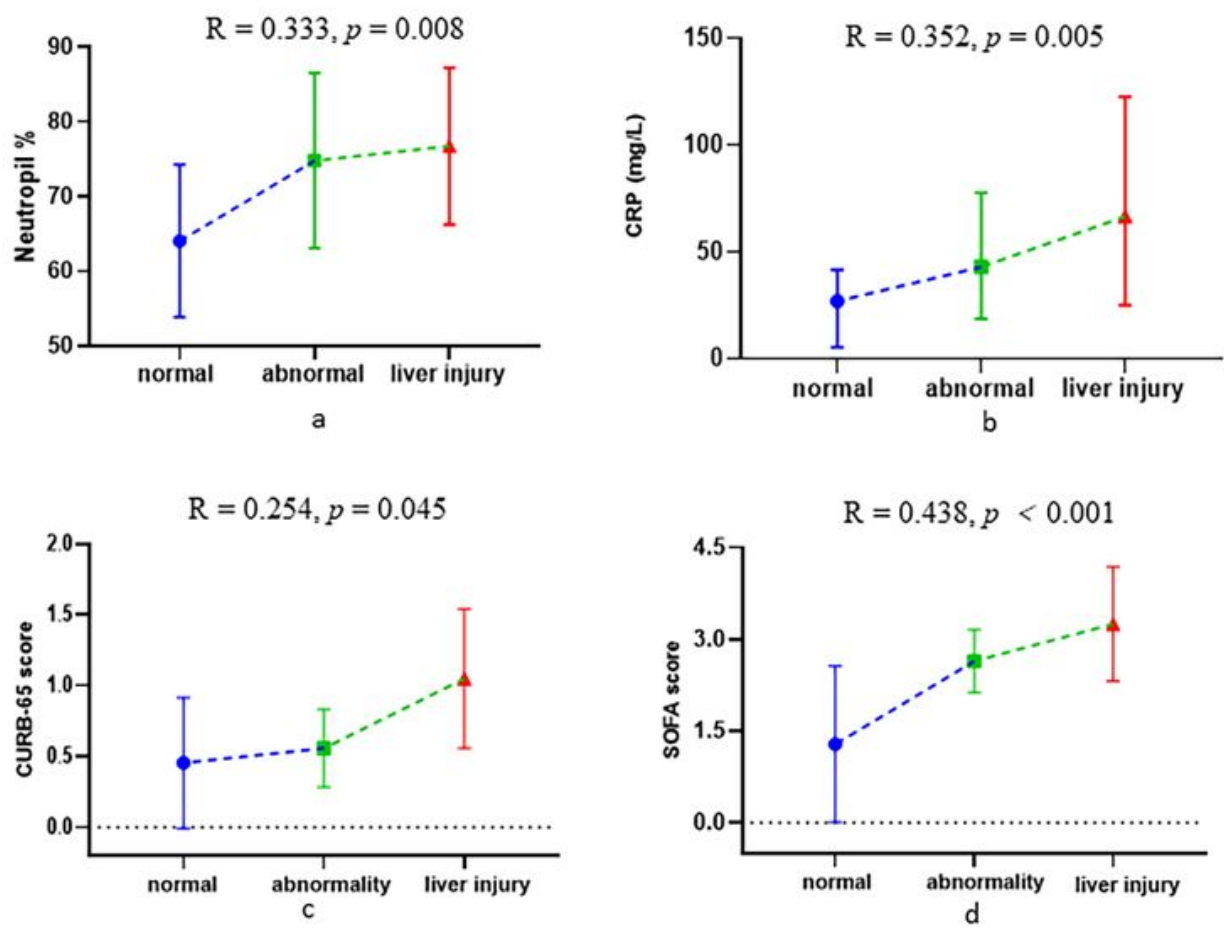

$\mathrm{R}=0.277, p=0.028$

$$
\mathrm{R}=0.374, p=0.004
$$
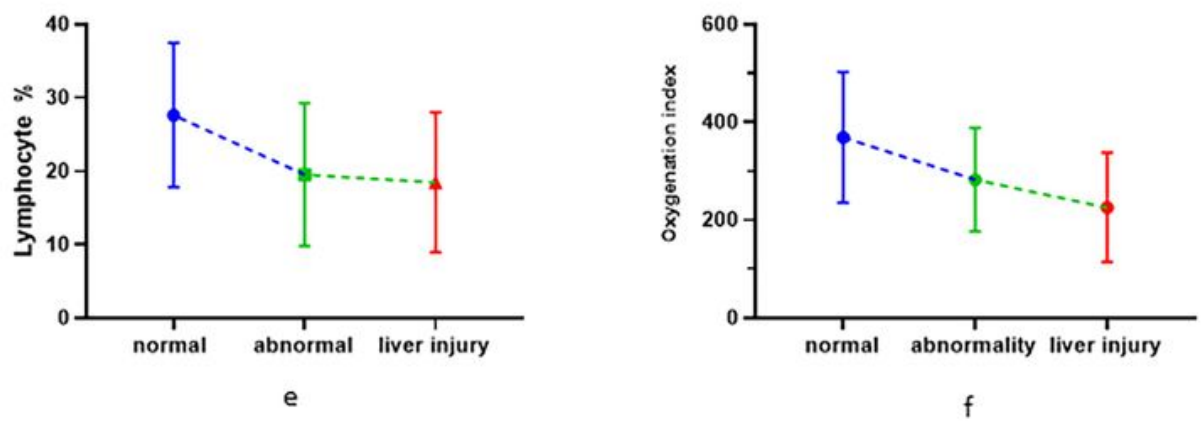

\section{Figure 4}

Caption: the correlation between the severity of liver injury and laboratory results. Fig. a: Description text: the correlation between the severity of liver injury and the percentage of neutrophil. Fig. b: Description text: the correlation between the severity of liver injury and CRP. Fig. c: Description text: the correlation between the severity of liver injury and CURB-65 score. Fig. d: Description text: the correlation between the severity of liver injury and SOFA score. Fig. e: Description text: the correlation between the severity of liver 
injury and the percentage of lymphocyte. Fig. f: Description text: the correlation between the severity of liver injury and oxygenation index.

\section{Supplementary Files}

This is a list of supplementary files associated with this preprint. Click to download.

- supplementalfigure11.tif

- supplementalfigure22.tif 